\title{
Involvement of haptoglobin in prevention of oxidative stress cause by hemoglobin in preeclampsia
}

\author{
Hagit Goldenstein, Nina Levy, Andrew Levy \\ Department of Anatomy and Cell Biology, Technion Faculty of Medicine, Technion Israel Institute of Technology, Haifa, Israel \\ Email:alevy@tx.technion.ac.il,ninal@techunix.technion.ac.il, hagitity@gmail.com
}

Received 13 August 2012; revised 17 September 2012; accepted 3 October 2012

\begin{abstract}
Haptoglobin (Hp) is a hemoglobin (Hb) binding protein which plays an important role in neutralizing oxidation reactions stimulated by heme-derived iron. Differences in Hp types due to the polymorphic nature of the gene have led to the discovery that individuals carrying the Hp 2-2 genotype are at increased risk of developing vascular complications in the setting of diabetes. Preeclampsia is a pregnancy related disease that is thought to be caused by increases in oxidative stress. The role of Hp polymorphism is determining preeclampsia has been addressed by several clinical studies but the results have been contradictory. Larger longitudinal studies are needed to answer this important question.
\end{abstract}

Keywords: Haptoglobin; Preeclampsia; Oxidative Stress; Hemoglobin; ROS

\section{INTRODUCTION}

Preeclampsia is a multi-factorial and multisystem disease of pregnancy that is a leading cause of maternal and perinatal morbidity and mortality. About 3\% - 8\% of all pregnancies are affected by it. Although the pathophysiology of this disease is not completely understood, recent evidence suggests that an increase in reactive oxygen species (ROS) may contribute to the etiology of the disease. Haptoglobin (Hp) is an acute-phase serum protein whose primary function is to bind extracellular hemoglobin and prevent heme-iron from participating in oxidative reactions. Since $\mathrm{Hp}$ is a polymorphic gene whose function is related to oxidative stress, it has been suggested that Hp genotype may influence the development of preeclampsia. This review will summarize the current knowledge of $\mathrm{Hp}$ and its possible role in preeclampsia as well as studies using antioxidants in the treatment thereof.

\section{PREECLAMPSIA}

The known risk factors for preeclampsia are kidney dis- ease, age over 35, obesity, diabetes, chronic hypertension, multi fetal pregnancy, and previous preeclampsia or fetal congenital abnormality. The risk of preeclampsia is two to fivefold higher in pregnant women if they have a family history of the disease [1]. Therefore, a genetic factor also exists. Preeclampsia is characterized by reduced placental perfusion due to increased peripheral vascular resistance and vasospasm and usually develops after 20 weeks of gestation. Preeclampsia is diagnosed using three concomitant parameters: hypertension over 140/90 mmHg, proteinuria of at least $300 \mathrm{mg}$ protein in a $24 \mathrm{~h}$ urine collection, and edema. Women with severe preeclampsia show other symptoms such as renal and liver dysfunction as well as hematological, neurological and visual disturbances. One such complication is known as the HELLP syndrome (hemolysis, elevated liver enzymes, and low platelets). There is no cure for this disease except delivery of the baby. Therefore, up to $15 \%$ of preterm births are a result of preeclampsia.

\section{Pathophysiology of Preeclampsia}

Preeclampsia can be considered as a two stage disease. The first stage of preeclampsia is abnormal placentation. Inadequate invasion of the chorionic villi after implanttation causes increased uterine arterial resistance, in contrast to decreased resistance, as occurs in normal pregnancies. This decrease in uterine arterial resistance is essential for retaining blood flow to the fetus. Thus the increase in arterial resistance causes chronic placental ischemia that can lead to intrauterine growth retardation and fetal death. The second stage of preeclampsia, which is less well understood, involves development of maternal endothelial cell dysfunction, which is the main cause of all clinical symptoms.

There are a number of mechanisms that may contribute to endothelial cell dysfunction. First, chronic placental ischemia blocks normal progression of the electron transport chain and leads to build up of ROS which are released into the maternal circulation and can adversely affect endothelial cell function. The role of increased oxidative stress in preeclampsia will be dis- 
cussed more extensively below. Second, an inappropriate balance of angiogenic factors exists in women with preeclampsia as a result of placental ischemia. For example, in preeclampsia patients, the levels of soluble fms-like tyrosine kinase-1 (sFlt1), a VEGF antagonist, are elevated, leading to decreased free VEGF [2]. Thus, the role of VEGF in maintaining the integrity of the glomerular filtration barrier is impaired leading to decreased filtration in the glomeruli and increased proteinuria. Third, faulty invasion of the chorionic villi is thought to cause disruption of the placental barrier and release of necrotic trophoblastic tissue into the maternal circulation. Another abnormality involves deposition of lipid deposits in the wall of the spiral arteries. Both of these processes stimulate the maternal immune response, leading to increased levels of cytokines and immune cells which can adversely affect the maternal endothelium.

\section{OXIDATIVE STRESS IN PREGNANCY}

Oxidative stress results from decreased levels of antioxidants or increased levels of ROS followed by lipid peroxidation. Free radicals, such super oxide and hydroxyl radical can oxidize proteins and cell membranes which lead to their dysfunction. Under normal conditions free radicals are formed during cell metabolism but are immediately neutralized by intracellular antioxidants such as catalase, superoxide dismutase (SOD) and glutathione peroxidase or by extracellular antioxidants such as transferrin, albumin and lactoferrin in the plasma. Also dietary antioxidants such as vitamin E scavenge lipid soluble free radicals while vitamin $C$ scavenges water soluble free radicals [3].

Oxidative stress is known to increase during normal pregnancy. This increase evolves from increased metabolism and increased basal oxygen and energy consumption. The main organ that produces free radicals is the placenta, which is highly vascular and is rich in mitochondria and macrophages. These cell organelles produce large amount of oxidants that can damage the placenta. However, in order to prevent this damage antioxidant levels also increase. An increase in radical scavengers such as SOD, catalase, bilirubin, glutathione peroxidase and glutathione reductase has been shown. Also, there is an increase in uptake of vitamin C by an ATP-driven mechanism or by diffusion if the concentration is high enough. Vitamin E decreases during the course of pregnancy and can be augmented by oral supplementation. Therefore, in normal pregnancy, the elevated levels of oxidants are counter balanced by elevated levels of antioxidants, so no severe oxidative damage is done [3].

\section{Oxidative Stress in Preeclampsia}

Unlike normal pregnancy, in preeclampsia the antio- xidant levels are not sufficient to prevent the oxidative damage cause by elevated levels of free radicals. Moreover, some antioxidant levels and activities are decreased, such as vitamin E, glutathione peroxidase and SOD. SOD is important for preventing peroxynitrite formation from NO and superoxide. Because SOD levels are decreased, more peroxynitrite is formed. In addition to its role as a strong oxidant, peroxynitrite formation results in decreased levels of NO, which is important for vasodilatation. Another source of oxidative stress is leukocyte activation, resulting in increased activity of NADPH oxidase and production of pro-inflammatory cytokines such as TNF- $\alpha$, IL-6 and VCAM- 1 . The combined effect of all of these factors is an increase in lipid peroxidation, endothelial cell dysfunction and vasospasm [3].

\section{HAPTOGLOBIN}

Haptoglobin (Hp) is a plasma protein, which is known best as a hemoglobin binding protein. It is an acute phase protein and thus its transcription is increased due to some inflammatory stimuli, such as IL6, IL1 and TNF. Hp gene is located on chromosome 16q22 and has two major classes of alleles in humans-1 and 2. As a result three different genotypes can be formed: homozygous (1-1 or 2-2) and heterozygous (2-1). Hp allele 1 is common to human and animals, but allele 2 is unique to humans, and was created during evolution from an unequal crossing over between two Hp 1 alleles. Allele 2 has a duplication of exons 3 and 4 of allele 1 . This duplication leads to differences in the polymeric structure of the protein. Hp 1-1 is a dimer, Hp 2-1 is a linear polymer and Hp 2-2 is a cyclic polymer [4]. Using gel electrophoresis these proteins can be identified due their unique patterns: Hp 1-1 shows one fast migrating band, Hp 2-2 shows series of slow migrating bands, and Hp 2-1 shows a weak fast migrating band and another series of slow migrating bands [5] (Figure 1).

Hp synthesis occurs mainly in the liver. Recent studies suggest that it is also produced by the endometrium and decidua cells and during pregnancy the levels of $\mathrm{Hp}$ increase [6].

$\mathrm{Hp}$ is synthesized as a single polypeptide chain, which being cleaved into $\alpha$ and $\beta$ chains that are joined by disulfide bonds. The Hp 1-1 and Hp 2-2 proteins have the same size $\beta$ chain (45 kD). However, due to the duplication event, they differ in $\alpha$ chain size, which is $9 \mathrm{kD}$ for Hp1 and $16 \mathrm{kD}$ for Hp 2 [4].

Due to positive selection during the evolution, allele 2 became more common in the northwestern European population, which may be related to its superior function as an antibacterial agent. The prevalence of the Hp 1-1, 2-2 and 2-1 genotypes in most western countries is $16 \%$, $36 \%$ and $48 \%$ respectively. However, there is consider- 


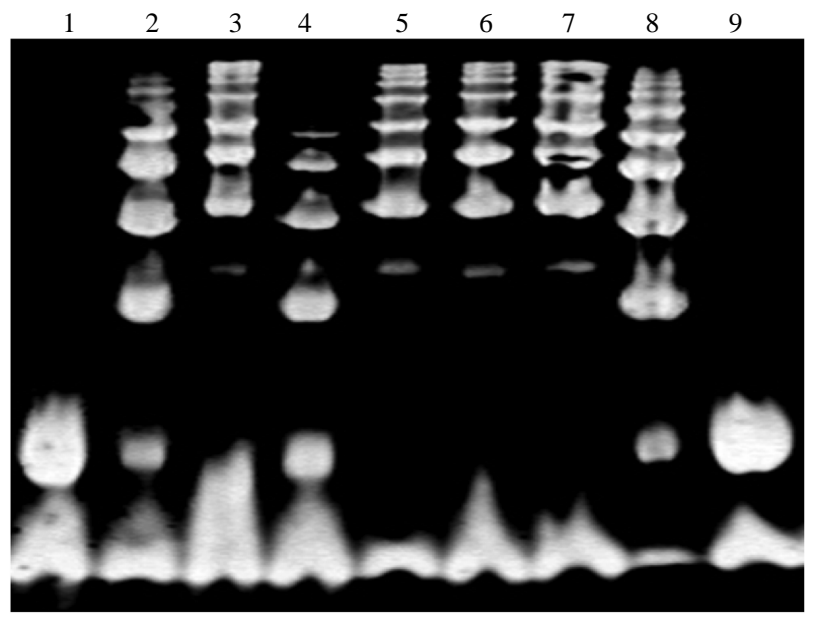

Figure 1. Hp typing of hemoglobin enriched serum by gel electrophoresis. Each Hp type has a characteristic banding pattern. Lanes 1 and 9 are Hp 1-1, lanes 2, 4, and 8 are Hp 2-1, and 3, 5, 6 and 7 are Hp 2-2 [4].

able variability in the prevalence of the three Hp types in other geographic areas. For example, in Southeast Asia, about $90 \%$ of all individuals have Hp 2-2 genotype [5].

\subsection{Hp Biological Functions}

\subsubsection{Antioxidant Role}

The main function of $\mathrm{Hp}$ is to bind $\mathrm{Hb}$ and to form a Hp$\mathrm{Hb}$ complex. Free $\mathrm{Hb}$ is toxic to the cell membrane and to plasma proteins due to its oxidative potential. By binding to $\mathrm{Hb}, \mathrm{Hp}$ serves as an anti-oxidant, and prevents $\mathrm{Hb}$ from interacting with other components to yield free radicals [4]. As part of the Hp-Hb complex Hp stabilizes the iron in the heme pocket of $\mathrm{Hb}$ and prevents its participation in oxidative reactions. Previous research showed that Hp 1-1 is more effective than Hp 2-2 in preventing Hb-induced oxidation of plasma components [7].

$\mathrm{Hb}-\mathrm{Hp}$ complexes are cleared in the liver by binding to CD163, a receptor on Kupffer cells. In this manner, iron loss in the urine and renal oxidative damage is prevented [4]. It was found that $\mathrm{Hp} 2-2-\mathrm{Hb}$ complexes are cleared more slowly than Hp 1-1-Hb complexes, which may allow the $\mathrm{Hb}$ which is complexed with $\mathrm{Hp}$ 2-2 more opportunity to react with oxidizing agents [8]. One important molecule that can bind to the $\mathrm{Hp} / \mathrm{Hb}$ complex is nitric oxide (NO) which is important in maintaining vascular tone, especially in the uterine arteries. In Hp 2-2 individuals the $\mathrm{Hp} / \mathrm{Hb}$ complex binds and deoxygenates NO, resulting in its decreased availability [4].

\subsubsection{Angiogenesis}

Hp is as an angiogenic factor and is important in the proliferation and differentiation of endothelial cells. It was found that Hp 2-2 has greater angiogenic potency than Hp 1-1. Hp has other important roles, such as antiin- flammatory effects, regulator of leukotrienes and prostaglandins, and immunomodulation that will not be discussed here [4].

\subsubsection{Hp in Diabetes Mellitus}

Due to the role of $\mathrm{Hp}$ as an antioxidant, its function is crucial under conditions of oxidative stress and hemolysis. A common disease in which oxidative stress and intravascular hemolysis are increased is Diabetes Mellitus (DM). High levels of glucose cause the $\mathrm{Hb}$ in $\mathrm{Hb}-\mathrm{Hp}$ complexes to be more redox active, and thus can undergo oxidation reactions more easily [4].

In the Strong Heart Study, a three- to fivefold increased risk of CVD was found in type 2 diabetic individuals with Hp 2-2 phenotype [9]. Similar results were found in the EDC study which prospectively followed individuals with type 1 diabetes, where a twofold increased risk in CVD was seen in Hp 2-2 individuals [10]. Numerous additional investigations have extended this finding to include approximately 8 - 10 thousand diabetic subjects of varying ethnic backgrounds [10-13]. These findings have now established that Hp 2-2 genotype is a risk factor for cardiovascular disease in the setting of diabetes.

Hp 2-2 phenotype has also been found to be associated with a more rapid decline in renal function in diabetes patients, and therefore can be a marker for predicting the risk of developing end stage renal disease [14]. Other vascular complications of diabetes such as retinopathy and stroke have been studied and shown to be associated with the Hp 2-2 genotype [15,16]. Gestational diabetes (GDM) and Hp type has also been investigated. In a study involving 250 Caucasian pregnant women it was found that GDM was significantly increased in women with Hp 2-2 and Hp 2-1 phenotypes [17]. In summary, these studies show that the Hp 2 allele is associated with increased multi-organ vascular complications in the setting of diabetes.

\subsubsection{Hp and Hypertension}

There have been conflicting reports regarding the role of Hp polymorphism in hypertension. A Brazilian cross sectional study investigated the relationship between cardiovascular disease in diabetics and Hp phenotype. Although no association between CVD and Hp genotype was identified, there was an approximately four fold increase in refractory hypertension (RH) in type 2 diabetics with Hp type 2-1 [18]. In a previous study on hypertension, an association between Hp 2-2 phenotype and higher occurrence of RH was found [19]. A second recent Brazilian study showed that individuals of the $\mathrm{Hp}$ 1-1 phenotype had an approximately 3 fold increase in essential hypertension [20]. These conflicting reports may be due to the cross sectional nature of the studies 
and indicates that additional prospective studies need to be carried out.

\section{HAPTOGLOBIN AND PREECLAMPSIA}

As mention above, a possible cause for preeclampsia is oxidative stress. Therefore it was suspected that due to the superior role of Hp 1-1 as an anti-oxidant, the distribution of Hp 1-1 phenotype in preeclampsia might be lower than other phenotypes. This hypothesis has been checked in a case controlled study which included 120 women with preeclampsia and 240 healthy women. It was found that the prevalence of Hp 1-1 was significantly decreased among women with preeclampsia. In addition almost $50 \%$ of the women at first delivery with Hp 2-2 phenotype or Hp 2-1 phenotype developed preeclampsia while only $25 \%$ with the Hp 1-1 phenotype developed preeclampsia. Surprisingly, it was not found that the Hp 2-2 phenotype was more prevalent in women with severe preeclampsia. Furthermore, no statistically significant difference was established by comparing healthy women to women with early or severe pree-clampsia. The lack of statistical significance might be due to the insufficient sample size of the group. However, these data suggest that Hp 1-1 has a protective role in preventing the development of preeclampsia, possibly due to its role in preventing oxidative damage caused by free $\mathrm{Hb}$ [21].

However, in a study done by Depypere and associates, opposite results were found. In this prospective study 60 Caucasian women with preeclampsia were followed from the time that preeclampsia was diagnosed until delivery. Two hundred healthy women were chosen as a control group. The results from this study showed that the Hp 1-1 phenotype was significantly more prevalent (28\%) among women with preeclampsia than in healthy women (16\%). Also, more severe clinical symptoms were found in patients with Hp 1-1 phenotype. Higher diastolic blood pressure (DBP) and systolic blood pressure (SBP), and higher levels of proteinuria were found in Hp 1-1 women. Surprisingly, the lowest levels of proteinuria were found within patients with $\mathrm{Hp} \mathrm{2-2}$ phenotype [22].

An additional study which looked at the association of Hp polymorphism and the development of severe preeclampsia (with or without HELLP syndrome) found no statistically significant differences in the prevalence of disease among the different Hp genotypes [23].

The contradictory results from these studies might be explained by ethnic differences between the groups or possibly due to different screening criteria that were applied to the patient population. For example, one study excluded only women with chronic hypertensive disorder, while the other excluded pre-existing hypertension, morbid obesity, gestational diabetes or multifetal pregnancy.
The cross sectional nature of these studies may also introduce a bias in the patient population, making it difficult to compare between studies. In addition there is evidence that Hp phenotype may affect different aspects of pregnancy such as the development of gestational diabetes (as mentioned above) or the general level of fertility [24]. All of these confounding factors suggest that more carefully controlled longitudinal prospective studies involving adequate numbers of patients need to be performed in order to determine if there is a significant association between Hp phenotype and preeclampsia.

\section{TREATMENT OF PREECLAMPSIA WITH ANTIOXIDANTS}

A number of studies have looked at the effects of antioxidant treatment (vitamin $\mathrm{E}$ and $\mathrm{C}$ ) on the development of preeclampsia. While some studies have shown promising results [25,26], a large meta-analysis of 15 studies concluded that there is no evidence to support treatment of preeclampsia with vitamins $E$ and C [27]. This is similar to the results of a meta-analysis of vitamin $\mathrm{E}$ treatment of CVD over the years which did not show overall benefit [28]. However, a recent prospective study reported that vitamin $\mathrm{E}$ supplementation resulted in less CVD among diabetic Hp 2-2 patients compared to placebo controls [13]. These results suggest that in order to see benefit it may be necessary stratify patients according to antioxidant status. Interestingly, one trial of preeclampsia looked at the benefit of vitamin $\mathrm{E}$ and $\mathrm{C}$ in women who were diabetic (type I). While the overall results showed no significant benefit, there was a trend toward decreased disease in women with lower initial antioxidant status (lower serum vitamin E and C levels). These women may correspond the diabetic Hp 2-2 subgroup in the CVD study that suffer higher levels of oxidative stress compared to other Hp genotypes and may have reduced endogenous antioxidant capacity. It would be interesting to look at the benefit of antioxidant treatment in preeclamptic women stratified by Hp genotype.

\section{CONCLUSION AND FUTURE DIRECTIONS}

Preeclampsia is a severe pregnancy related disease with many complications and a high percent mortality. Therefore characterizing genetic risk factors that can identify women at higher risk will allow for greater supervision and treatment in order to prevent the development of the disease. Hp has been found to be associated with several diseases that are related to oxidative stress such as CVD, nephropathy, and retinopathy in the setting of diabetes. Due to its role as an antioxidant, Hp may play a critical role in the development of preeclampsia.

Several conflicting studies have been reported con- 
cerning the relationship between Hp genotype and prevalence of preeclampsia. The contradictions between the studies cited may be due to small sample size, different ethnic origins, and the examination of prevalent as opposed to incident disease. Therefore, in order to test this hypothesis it is necessary to study a large number of patients from different ethnic backgrounds and to look at longitudinal development of the disease. In addition, an animal model may be helpful in normalizing the genetic background of the participants and increasing the sample size. Transgenic mice carrying the Hp 2 allele already exist and are being used to study oxidative disease [29]. Finally, the possible beneficial effect of vitamin treatment on preeclampsia in women of defined Hp genotype should be determined. These studies might lead to finding a simple and inexpensive treatment for a disease that offers limited therapy and is cured only by premature delivery.

\section{REFERENCES}

[1] Uzan, J., Carbonnel, M., Piconne, O., Asmar, R. and Ayoubi, J.M. (2011) Pre-eclampsia: Pathophysiology, diagnosis, and management. Journal of Vascular Health and Risk Management, 7, 467-474.

[2] Maynard, S.E., Min, J.Y., Merchan, J., Lim, K.H., Li, J., Mondal, S., Libermann, T.A., Morgan, J.P., Sellke, F.W., Stillman, I.E., Epstein, F.H., Sukhatme, V.P. and Karumanchi, S.A. (2003) Excess placental soluble fms-like tyrosine kinase 1 (sFlt1) may contribute to endothelial dysfunction, hypertension, and proteinuria in preeclampsia. The Journal of Clinical Investigation, 111, 649-658.

[3] Siddiqui, I.A., Jaleel, A., Tamimi, W. and Al Kadri, H.M. (2010) Role of oxidative stress in the pathogenesis of preeclampsia. Archives of Gynecology and Obstetrics, 282, 469-474. doi:10.1007/s00404-010-1538-6

[4] Levy, A.P., Asleh, R., Blum, S., Levy, N.S., Miller-Lotan, R., Kalet-Litman, S., Anbinder, Y., Lache, O., Nakhoul, F.M., Asaf, R., Farbstein, D., Pollak, M., Soloveichik, Y.Z., Strauss, M., Alshiek, J., Livshits, A., Schwartz, A., Awad, H., Jad, K. and Goldenstein, H. (2010) Haptoglobin: Basic and clinical aspects. Antioxidants \& Redox Signaling, 12, 293-304. doi:10.1089/ars.2009.2793

[5] Langlois, M.R. and Delanghe, J.R. (1996) Biological and clinical significance of haptoglobin polymorphism in humans. Clinical Chemistry, 42, 1589-1600.

[6] Berkova, N., Lemay, A., Dresser, D.W., Fontaine, J.Y., Kerizit, J. and Goupil, S. (2001) Haptoglobin is present in human endometrium and shows elevated levels in the decidua during pregnancy. Molecular Human Reproduction, 7, 747-754. doi:10.1093/molehr/7.8.747

[7] Buehler, P.W., Abraham, B., Vallelian, F., Linnemayr, C., Pereira, C.P., Cipollo, J.F., Jia, Y., Mikolajczyk, M., Boretti, F.S., Schoedon, G., Alayash, A.I. and Schaer, D.J. (2009) Haptoglobin preserves the CD163 hemoglobin scavenger pathway by shielding hemoglobin from peroxidative modification. Blood, 113, 2578-2586. doi:10.1182/blood-2008-08-174466

[8] Asleh, R., Marsh, S., Shilkrut, M., Binah, O., Guetta, J., Lejbkowicz, F., Enav, B., Shehadeh, N., Kanter, Y., Lache, O., Cohen, O., Levy, N.S. and Levy, A.P. (2003) Genetically determined heterogeneity in hemoglobin scavenging and susceptibility to diabetic cardiovascular disease. Circulation Research, 92, 1193-1200. doi:10.1161/01.RES.0000076889.23082.F1

[9] Levy, A.P., Hochberg, I., Jablonski, K., Resnick, H.E., Lee, E.T., Best, L. and Howard, B.V. (2002) Haptoglobin phenotype is an independent risk factor for cardiovascular disease in individuals with diabetes: The strong heart study. Journal of the American College of Cardiology, 40, 1984-1990. doi:10.1016/S0735-1097(02)02534-2

[10] Costacou, T., Ferrell, R.E. and Orchard, T.J. (2008) Haptoglobin genotype: A determinant of cardiovascular complication risk in type 1 diabetes. Diabetes, 57, 1702-1706. doi:10.2337/db08-0095

[11] Roguin, A., Koch, W., Kastrati, A., Aronson, D., Schomig, A. and Levy, A.P. (2003) Haptoglobin genotype is predictive of major adverse cardiac events in the 1-year period after percutaneous transluminal coronary angioplasty in individuals with diabetes. Diabetes Care, 26, 2628-2631. doi:10.2337/diacare.26.9.2628

[12] Suleiman, M., Aronson, D., Asleh, R., Kapeliovich, M.R., Roguin, A., Meisel, S.R., Shochat, M., Sulieman, A., Reisner, S.A., Markiewicz, W., Hammerman, H., Lotan, R., Levy, N.S. and Levy, A.P. (2005) Haptoglobin polymorphism predicts 30-day mortality and heart failure in patients with diabetes and acute myocardial infarction. Diabetes, 54, 2802-2806. doi:10.2337/diabetes.54.9.2802

[13] Milman, U., Blum, S., Shapira, C., Aronson, D., Miller-Lotan, R., Anbinder, Y., Alshiek, J., Bennett, L., Kostenko, M., Landau, M., Keidar, S., Levy, Y., Khemlin, A., Radan, A. and Levy, A.P. (2008) Vitamin E supplementation reduces cardiovascular events in a subgroup of middle-aged individuals with both type 2 diabetes mellitus and the haptoglobin 2-2 genotype: A prospective double-blinded clinical trial. Arteriosclerosis, Thrombosis, and Vascular Biology, 28, 341-347. doi:10.1161/ATVBAHA.107.153965

[14] Costacou, T., Ferrell, R.E., Ellis, D. and Orchard, T.J. (2009) Haptoglobin genotype and renal function decline in type 1 diabetes. Diabetes, 8, 2904-2909. doi:10.2337/db09-0874

[15] Levy, A.P., Roguin, A., Hochberg, I., Herer, P., Marsh, S., Nakhoul, F.M. and Skorecki, K. (2000) Haptoglobin phenotype and vascular complications in patients with diabetes. The New England Journal of Medicine, 343, 969970. doi:10.1056/NEJM200009283431313

[16] Nakhoul, F.M., Zoabi, R., Kanter, Y., Zoabi, M., Skorecki, K., Hochberg, I., Leibu, R., Miller, B. and Levy, A.P. (2001) Haptoglobin phenotype and diabetic nephropathy. Diabetologia, 44, 602-604. doi:10.1007/s001250051666

[17] Mustafa, S., Vukovich, T., Prikoszovich, T., Winzer, C., Schneider, B., Esterbauer, H., Wagner, O. and KautzkyWiller, A. (2004) Haptoglobin phenotype and gestational diabetes. Diabetes Care, 27, 2103-2107. doi:10.2337/diacare.27.9.2103 
[18] Wobeto, V.P., Pinho Pda, C., Souza, J.R., Zaccariotto, T.R. and Zonati Mde, F. (2011) Haptoglobin genotypes and refractory hypertension in type 2 diabetes mellitus patients. Arquivos Brasileiros de Cardiologia, 97, 338-345. doi:10.1590/S0066-782X2011005000107

[19] Delanghe, J.R., Duprez, D.A., De Buyzere, M.L., Bergez, B.M., Claeys, L.R., Leroux-Roels, G.G. and Clement, D.L. (1995) Refractory hypertension is associated with the haptoglobin 2-2 phenotype. Journal of Cardiovascular Risk, 2, 131-136. doi:10.1097/00043798-199504000-00008

[20] Miranda-Vilela, A.L., Akimoto, A.K., Alves, P.C., Ferreira, L.B., Lordelo, G.S., Melo, J.G., Grisolia, C.K., Oliveira, S.F. and Klautau-Guimaraes, M.N. (2010) Evidence for an association between haptoglobin and MnSOD (Val9Ala) gene polymorphisms in essential hypertension based on a Brazilian case-control study. Genetics and Molecular Research, 9, 2166-2175. doi:10.4238/vol9-4gmr973

[21] Sammour, R.N., Nakhoul, F.M., Levy, A.P., Miller-Lotan, R., Nakhoul, N., Awad, H.R., Gonen, R. and Ohel, G. (2010) Haptoglobin phenotype in women with preeclampsia. Endocrine, 38, 303-308. doi:10.1007/s12020-010-9392-7

[22] Depypere, H.T., Langlois, M.R., Delanghe, J.R., Temmerman, M. and Dhont, M. (2006) Haptoglobin polymorphism in patients with preeclampsia. Clinical Chemistry and Laboratory Medicine, 44, 924-928. doi:10.1515/CCLM.2006.182

[23] Raijmakers, M.T., Roes, E.M., Te Morsche, R.H., Steegers, E.A. and Peters, W.H. (2003) Haptoglobin and its association with the HELLP syndrome. Journal of Medical Genetics, 40, 214-216. doi:10.1136/jmg.40.3.214
[24] Bottini, N., Gimelfarb, A., Gloria-Bottini, F., La Torre, M., Lucarelli, P. and Lucarini, N. (1999) Haptoglobin genotype and natural fertility in humans. Fertility and Sterility, 72, 293-296. doi:10.1016/S0015-0282(99)00210-1

[25] Chappell, L.C., Seed, P.T., Briley, A.L., Kelly, F.J., Lee, R., Hunt, B.J., Parmar, K., Bewley, S.J., Shennan, A.H., Steer, P.J. and Poston, L. (1999) Effect of antioxidants on the occurrence of pre-eclampsia in women at increased risk: A randomised trial. Lancet, 354, 810-816.

[26] Beazley, D., Ahokas, R., Livingston, J., Griggs, M. and Sibai, B.M. (2005) Vitamin C and E supplementation in women at high risk for preeclampsia: A double-blind, placebo-controlled trial. American Journal of Obstetrics \& Gynecology, 192, 520-521. doi:10.1016/j.ajog.2004.09.005

[27] Rossi, A.C. and Mullin, P.M. (2011) Prevention of preeclampsia with low-dose aspirin or vitamins $\mathrm{C}$ and $\mathrm{E}$ in women at high or low risk: A systematic review with meta-analysis. European Journal of Obstetrics \& Gynecology and Reproductive Biology, 158, 9-16. doi:10.1016/j.ejogrb.2011.04.010

[28] Miller, E.R. 3rd, Pastor-Barriuso, R., Dalal, D., Riemersma, R.A., Appel, L.J. and Guallar, E. (2005) Meta-analysis: High-dosage vitamin E supplementation may increase all-cause mortality. Annals of Internal Medicine, 142, $37-$ 46.

[29] Levy, A.P., Levy, J.E., Kalet-Litman, S., Miller-Lotan, R., Levy, N.S., Asaf, R., Guetta, J., Yang, C., Purushothaman, K.R., Fuster, V. and Moreno, P.R. (2007) Haptoglobin genotype is a determinant of iron, lipid peroxidation, and macrophage accumulation in the atherosclerotic plaque. Arteriosclerosis, Thrombosis, and Vascular Biology, 27, 134-140. doi:10.1161/01.ATV.0000251020.24399.a2 